\title{
Evaluation of lymph node metastasis in a rabbit tumor model: correlations between contrast-enhanced ultrasound and pathologic findings
}

\author{
Shin-Woo Ha ${ }^{1,2}$, Hyun-Je Lee ${ }^{3}$, Ae-Sin Cho ${ }^{1}$, Sung II Hwang ${ }^{1}$, Hak Jong Lee ${ }^{1,2,3}$ \\ 'Department of Radiology, Seoul National University Bundang Hospital, Seoul National \\ University College of Medicine, Seongnam; ${ }^{2}$ Research Institute, IMGT Co., Ltd., Seongnam; \\ ${ }^{3}$ Department of Nanoconvergence, Seoul National University Graduate School of \\ Convergence Science and Technology, Suwon, Korea
}

Purpose: The purpose of this study was to evaluate the ability of contrast-enhanced ultrasonography (CEUS) with microbubbles to detect metastatic lymph nodes (LNs) for treatment planning and prognosis.

Methods: For the metastatic LN model, ground $\mathrm{VX}_{2}$ tumor tissues were injected subcutaneously in 12 rabbits, just below the right hind limb. The rabbits were classified into three groups based on the LN area: group $A\left(n=4,>1.9 \mathrm{~cm}^{2}\right)$, group $B\left(n=4,1-1.9 \mathrm{~cm}^{2}\right)$, and group $C(n=4,<1$ $\left.\mathrm{cm}^{2}\right)$. The LNs were monitored on CEUS for 10 seconds after injecting $2.5 \mathrm{~mL}$ of microbubbles. The percent area of metastatic LNs was calculated on pathologic images and compared with CEUS images.

Results: In group A, the mean percent area of metastasis was $40.7 \% \pm 19.4 \%$. In all cases of metastasis, round-shaped perfusion defects were clearly observed in CEUS images. The metastatic areas were strongly correlated with pathologic findings. The mean percent area in group B was $21.5 \% \pm 14.4 \%$. The CEUS findings showed multiple nodular perfusion defects, clearly revealing the metastatic areas. In group B, the CEUS and pathologic findings were concordant for three of the four cases. The mean percent area in group $C$ was $9.1 \% \pm 6.4 \%$. However, in this group, CEUS only detected a small perfusion defect in one case.

Conclusion: CEUS has the potential to depict characteristic imaging features of metastatic LNs but still has limitations in early detection.

Keywords: Lymph nodes; Lymphatic metastasis; Microbubbles; Ultrasonography; Diagnostic imaging

\section{Introduction}

The lymphatic system is known to be involved in cancer metastasis (the spread of cancer from the primary tumor to other organs), and our understanding of the mechanisms of metastasis continues to evolve. Lymphatic vessels, which reach regional lymph nodes (LNs), are a possible metastatic route for

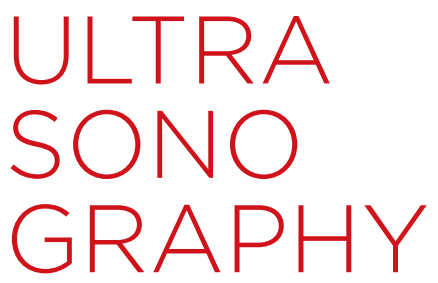

ORIGINAL ARTICLE

https://doi.org/10.14366/usg. 19010 pISSN: 2288-5919 - elSSN: 2288-5943 Ultrasonography 2020;39:60-69

Received: January 30, 2019

Revised: May 5, 2019

Accepted: June 10, 2019

Correspondence to: Hak Jong Lee, MD, PhD, Department of Radiology, Seoul National University Bundang Hospital, Seoul National University College of Medicine, 82 Gumi-ro 173beon-gil, Bundang-gu, Seongnam 13620, Korea

Tel. +82-31-787-7605

Fax. +82-31-787-8709

E-mail: hakjlee@snu.ac.kr

This is an Open Access article distributed under the terms of the Creative Commons Attribution NonCommercial License (http://creativecommons.org licenses/by-nc/4.0/) which permits unrestricted noncommercial use, distribution, and reproduction in any medium, provided the original work is properly cited.

Copyright (C) 2020 Korean Society of Ultrasound in Medicine (KSUM)

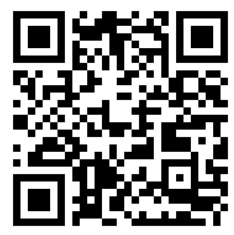

How to cite this article:

Ha SW, Lee HJ, Cho AS, Hwang SI, Lee HJ. Evaluation of lymph node metastasis in a rabbit tumor model: correlations between contrast-enhanced ultrasound and pathologic findings. Ultrasonography. 2020 Jan;39(1):6069. 
cancer cells [1]. LNs act as barriers that temporarily block the further spread of cancer cells. Once malignant metastatic cells spread and are observed in the LNs, current medical treatment options may be insufficient to provide a complete cure, resulting in an increased risk of mortality [2]. Therefore, the diagnosis of metastatic LNs is crucial for treatment planning, which can include surgery, radiation therapy, or adjuvant chemotherapy. Numerous methods have been used clinically to diagnose LN metastasis, including lymphoscintigraphy, computed tomography (CT), single-photon emission $\mathrm{CT}$, near-infrared fluorescence imaging, photoacoustic imaging, magnetic resonance imaging, and ultrasonography (US) [3]. These modalities provide sufficient information for clinicians and surgeons to decide on a treatment method, but some have limitations for intraoperative use, such as a requirement to transfer the patient to an imaging facility and a relatively long preparation time. US has several advantages over alternative techniques such as positron emission tomography/ CT and lymphoscintigraphy, including the absence of radiation exposure or other harmful effects, easy accessibility, and lower costs $[4,5]$.

Various modes of US exist, including as B-mode and color Doppler. Contrast-enhanced US (CEUS) combines traditional US with an ultrasound contrast agent [6]. CEUS images are generated from differences in acoustic impedance between soft tissues and gas in the ultrasound contrast agent, which contains commercial microbubbles (MBs). MBs are micron-sized bubbles (1-10 $\mu \mathrm{m})$ filled with non-toxic and safe gas (sulfur hexafluoride, SF6) that have been developed in various formulations to enhance contrast, circulation time, and stability since Gramiak and Shah [7] first showed that they improved contrast in echocardiography in the 1960s. Because MBs are administered intravenously, the applicability of MBs depends on the vascularity of specific organs or tumors. Some attempts have been made to visualize sentinel LNs (SLNs) in animal models and breast cancer patients using CEUS imaging [8-12]. To enhance diagnostic accuracy and facilitate intraoperative imaging, it is necessary to compare CEUS images with pathologic images from the same region. However, to our knowledge, few reports have conducted a comparative analysis of 1-to-1-matched CEUS and pathologic images.

In this study, with the ultimate goal of leveraging our findings for application in clinical practice, we utilized a US imaging modality and commercial MBs (SonoVue) to identify micro-metastases in popliteal LNs metastasized from $\mathrm{VX}_{2}$ tumors on the thigh in a rabbit model.

\section{Materials and Methods}

\section{Preparation of Animal Model}

This study was approved by the institutional animal care and use committee of our hospital. Twelve adult New Zealand white rabbits, weighing 2.0 to $2.5 \mathrm{~kg}$, were maintained in rooms with a constant temperature $\left(23^{\circ} \mathrm{C} \pm 2^{\circ} \mathrm{C}\right)$ and a 12 -hour light/dark cycle. Throughout the study period (December 2015 to February 2017), the VX tumor strain was maintained in the hind limb of a carrier rabbit through deep intramuscular injection [13-15]. To establish a model of the $\mathrm{VX}_{2}$ tumor and metastatic LNs, fresh tumor tissues without signs of necrosis were harvested and collected. Then, the collected tumor tissues were finely ground and subcutaneously injected into the other rabbits, just below the right hind limb, to induce $\mathrm{VX}_{2}$ tumor establishment and popliteal LN metastasis. After inoculation, the tumor and popliteal LNs were monitored. In our study, the early stage of LN metastasis (group C) was normally examined on the 10th day, the growth stage (group B) on the 14th day, and the advanced stage (group A) on the 21st day after inoculation.

\section{US Contrast Agent and Instrument}

The US contrast agent, SonoVue (Bracco, Milan, Italy), was reconstituted according to the manufacturer's instructions. Its size and morphology were characterized by Zetasizer (Nano ZS, Malvern Instruments, Malvern, UK) and an upright optical microscope (BX63, Olympus, Tokyo, Japan). Fig. 1 shows the shape and size of the MBs. Their average size was $2.2 \pm 0.6 \mu \mathrm{m}$ by the dynamic light scattering technique, with a broad size distribution, ranging from a few hundred nanometers to $10 \mu \mathrm{m}$.

Gray-mode US imaging and CEUS were performed using a clinical US system (iU22, Philips Healthcare, Best, The Netherlands) with a L12-5 broadband linear transducer. To reduce MB destruction, low mechanical index values were applied for imaging (0.2-0.4).

Before the US examinations, animals were anesthetized with respiratory anesthetic (isoflurane, Hana Pharm, Seoul, Korea). Rabbits were placed in the supine position, and the hair around the tumor and popliteal area was shaved to minimize differences in acoustic impedance between the US probe and the skin.

Before injection of the contrast agent, conventional US was performed in an attempt to identify the $\mathrm{VX}_{2}$ tumor and adjacent $\mathrm{LNs}$. Then, $2.5 \mathrm{~mL}$ of SonoVue solution was administered subcutaneously in the right foot. The injection was performed slowly in an attempt to avoid the rupture of MBs via high pressure during the injection. The injection site was massaged for approximately 1 minute to accelerate the movement of MBs into the lymphatic channels and LNs. Then, CEUS was performed in the popliteal area in an attempt to identify the metastatic LNs in the right leg and the normal LNs in 


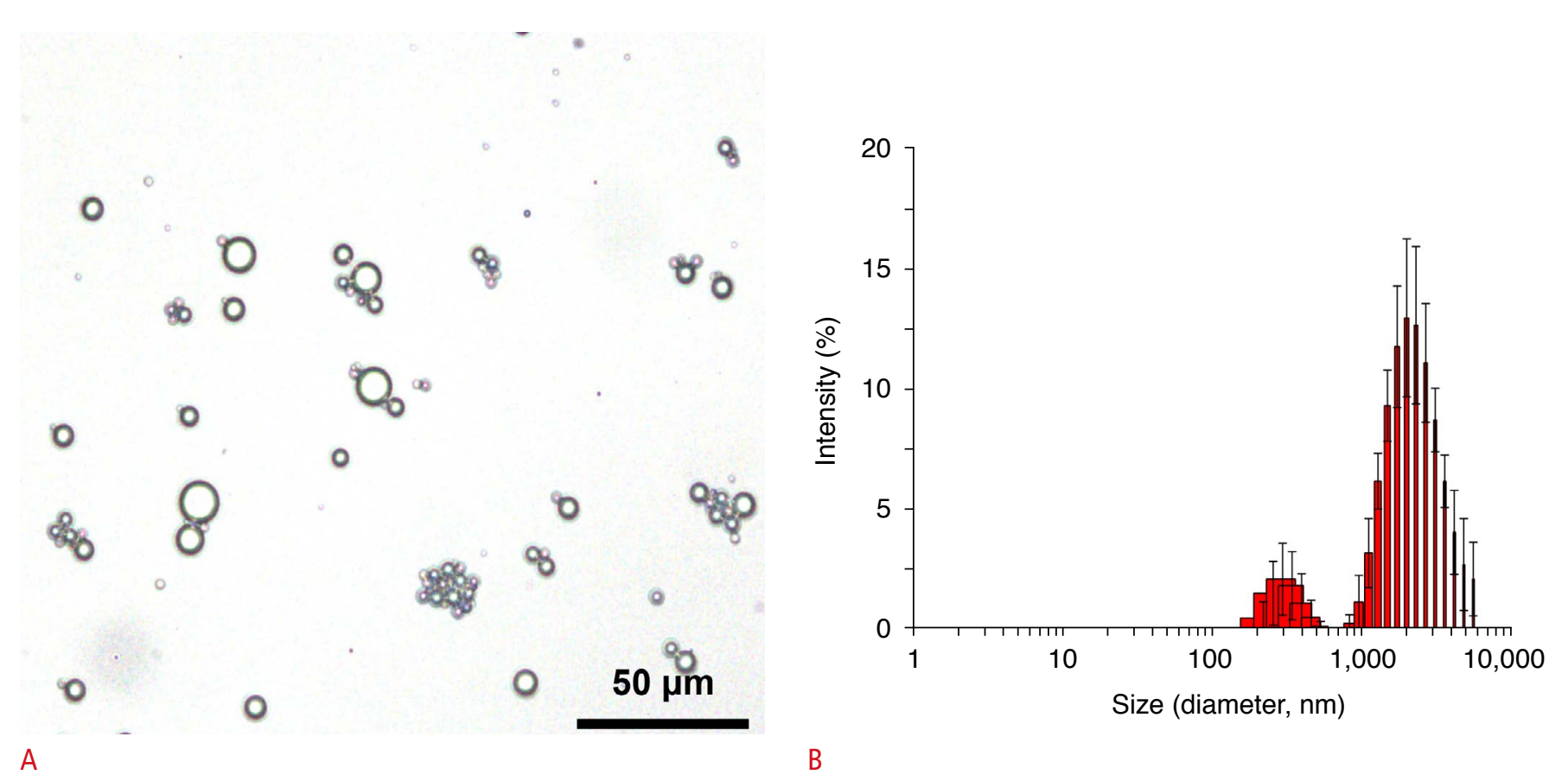

Fig. 1. The morphology and size of microbubbles.

A. A microscopic image shows variably-sized microbubbles. B. Dynamic light scattering analysis revealed that the sizes of microbubbles ranged from few hundred nanometers to $10 \mu \mathrm{m}$, with a mean size of $2.2 \pm 0.6 \mu \mathrm{m}$.

the contralateral leg. The patterns of enhancement of the LNs were observed and recorded. After the CEUS study, the metastatic LNs on the right leg and the control LNs on the contralateral leg were harvested and prepared using standard hematoxylin and eosin (H\&E) staining for histopathologic examinations.

\section{Quantitative Image Analysis}

Two radiologists with over 15 years of experience in US reviewed the video clips showing the CEUS examination of the LNs without knowledge of group allocation. They were asked to reach a consensus on the status of LNs as either positive or negative according to the presence or absence of perfusion defects in them.

For a quantitative analysis of LN metastasis, the tissue slides after H\&E staining were scanned using a slide scanner (Super Cool Scan, Nikon, Tokyo, Japan) in the TIFF format. The scanned images were uploaded into the Image J program version 1.4.3.67, which is freely available for download from the website of the National Institutes of Health. Under the supervision of a pathologist, the outlines of the LNs and metastatic lesions were drawn manually. The size and area of the LNs and metastatic lesions were measured. The percentage of metastatic areas in the LN was calculated as (metastatic area $\left[\mathrm{cm}^{2}\right] /$ $\mathrm{LN}$ area $\left.\left[\mathrm{cm}^{2}\right] \times 100\right)$. The percentage of areas with perfusion defects was also measured and calculated using the formula [(area of gray image-area of CEUS image)/area of gray image $\times 100$. To indicate the results of the 1-to-1 matching of the images, positive $(+)$ and negative (-) signs are presented in Table 1, representing identical or different diagnoses between the histologic and ultrasonic analyses, respectively.

\section{Statistical Analysis}

The animals were divided into three groups by the metastatic LN area on the right leg based on the pathologic specimens: group $A$ $(n=4)$, over $1.9 \mathrm{~cm}^{2}$; group $B(n=4)$, between 1 and $1.9 \mathrm{~cm}^{2}$; and group $C(n=4)$, less than $1 \mathrm{~cm}^{2}$. In each group, the length, width, and area of LNs; the metastatic area in the LNs; the percent area of metastasis; and the perfusion defects in the LNs were analyzed and compared using the Kruskal-Wallis and Mann-Whitney $\mathrm{U}$ tests. Moreover, the length of the normal LNs on the contralateral leg was measured and compared using the same statistical tests. In all statistical analyses, a two-tailed P-value of less than 0.05 was considered to indicate a statistically significant difference. Statistical analyses were performed using SPSS version 19.0 for Windows (IBM Corp., Armonk, NY, USA).

\section{Results}

\section{Categorization of LNs}

We investigated whether a higher metastatic stage was correlated 
LN

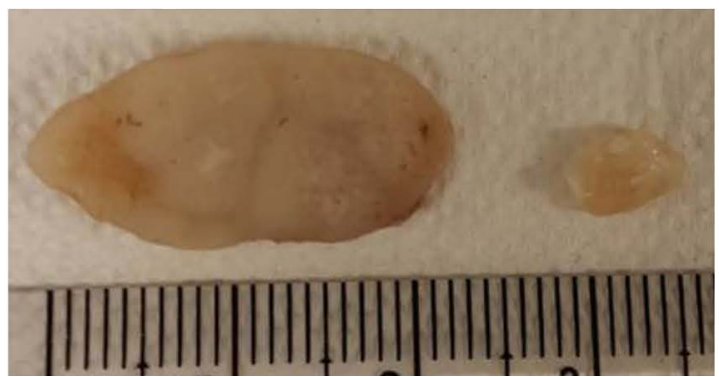

Group A

A

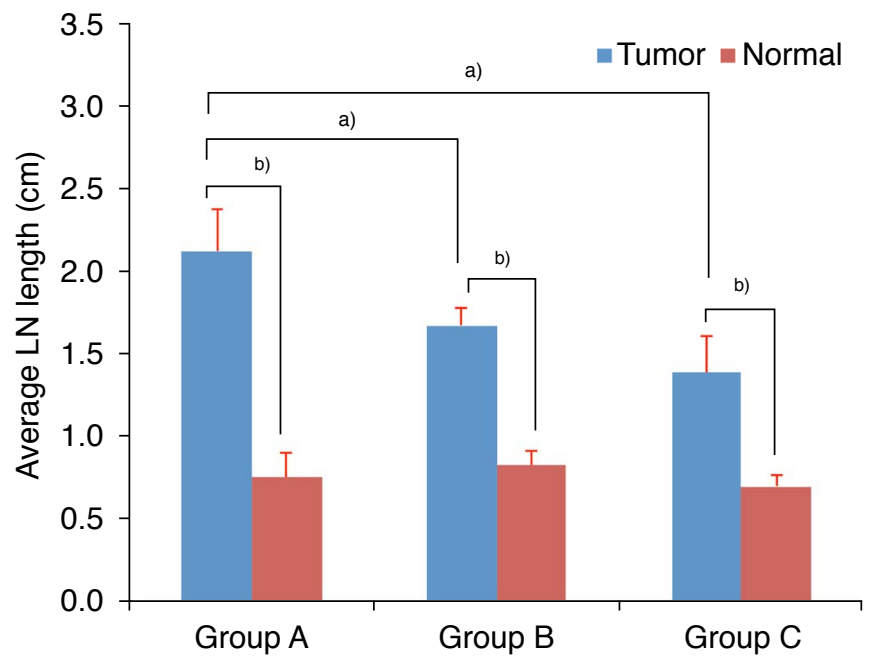

B

to $L N$ size. LNs were classified by the size of the metastatic LN. Fig. 2A shows representative pictures of both metastatic (left) and normal (right) LNs in each group. Fig. $2 B$ is a bar graph of the average LN size, and Table 1 shows the sizes of all LNs and tumors.

The mean length of the metastatic LNs in each group was $1.99 \pm 0.51 \mathrm{~cm}$ (range, 1.85 to $2.20 \mathrm{~cm}$ ) in group $A, 1.32 \pm 0.33 \mathrm{~cm}$ (range, 1.50 to $1.13 \mathrm{~cm}$ ) in group $B$, and $1.15 \pm 0.12 \mathrm{~cm}$ (range, 0.90 to $1.50 \mathrm{~cm}$ ) in group $C$. The mean width of the metastatic $L N s$ in each group was $1.42 \pm 0.32 \mathrm{~cm}$ (range, 1.02 to $1.70 \mathrm{~cm}$ ) in group $A$, $0.92 \pm 0.17 \mathrm{~cm}$ (range, 0.75 to $1.14 \mathrm{~cm}$ ) in group $B$, and $1.04 \pm 0.04$ $\mathrm{cm}$ (range, 0.90 to $1.50 \mathrm{~cm}$ ) in group $C$. The length of the metastatic LNs in each group was significantly different $(P=0.019$, KruskalWallis test). A paired group analysis revealed that there was a statistically significant difference between groups $A$ and $B(P=0.021$, Mann-Whitney test), as well as between groups $A$ and $C(P=0.021$, Mann-Whitney $U$ test). The width of the metastatic LNs did not show any statistically significant differences $(P=0.073$, Kruskal-Wallis test). The mean width of the normal $L N s$ on the contralateral leg was $0.75 \pm 0.19 \mathrm{~cm}$ (range, 0.63 to $0.90 \mathrm{~cm}$ ) in group $A, 0.80 \pm 0.08$
$\mathrm{LN}$

$\mathrm{CL}$

LN

$\mathrm{CL}$

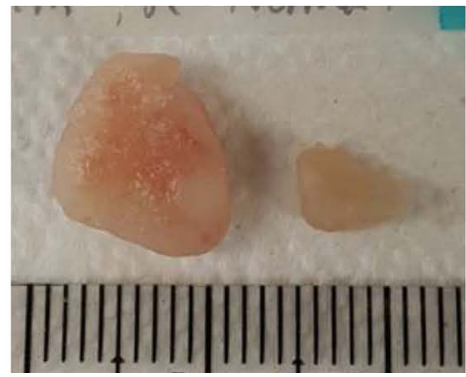

Group C
Fig. 2. Gross specimen showing metastatic and normal lymph nodes (LNs) in each group and a graph showing the LN length.

A. Gross specimen shows metastatic and normal LNs in each group. $\mathrm{CL}$, control. B. The size of the metastatic and normal LNs was statistically significantly different in each group. ${ }^{a} \mathrm{P}<0.05$ relative to tumor of group $A .{ }^{b} P<0.005$ relative to tumor of each group by Student's t test. $\mathrm{cm}$ (range, 0.69 to $0.90 \mathrm{~cm}$ ) in group $B$, and $0.58 \pm 0.10 \mathrm{~cm}$ (range, 0.50 to $0.71 \mathrm{~cm}$ ) in group $C$. The size of the normal LNs showed no statistically significant difference among the three groups ( $P=0.078$, Kruskal-Wallis test).

\section{CEUS Findings of the Metastatic LNs}

The metastatic LNs were observed by a clinical US system using CEUS, and visualized as perfusion defects within normally-enhancing tissues. Figs. 3-5 show representative findings of metastatic LNs seen on CEUS in each group.

Table 1 also shows the percent area of metastasis and perfusion defects in the LNs. All cases in group A were over $1.9 \mathrm{~cm}^{2}$ in area, and the mean area percentage of metastasis was $40.7 \% \pm 19.4 \%$. The percent area of perfusion defects was $74.5 \% \pm 9.8 \%$. All cases of metastasis were clearly visualized as round perfusion defects on CEUS (Figs. 3, 6). Group B was categorized as having an LN area between $1.0 \mathrm{~cm}^{2}$ and $1.9 \mathrm{~cm}^{2}$, and the mean percent area of metastasis was $21.5 \% \pm 14.4 \%$. The percent area of perfusion defects was $42.3 \% \pm 9.7 \%$. The CEUS findings showed multiple 


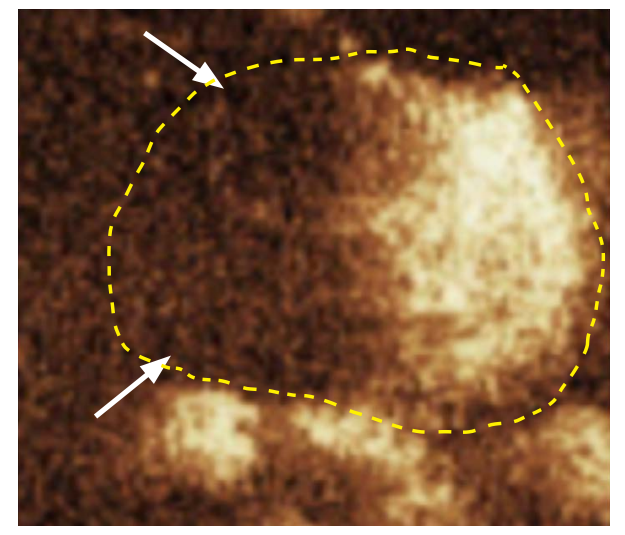

A

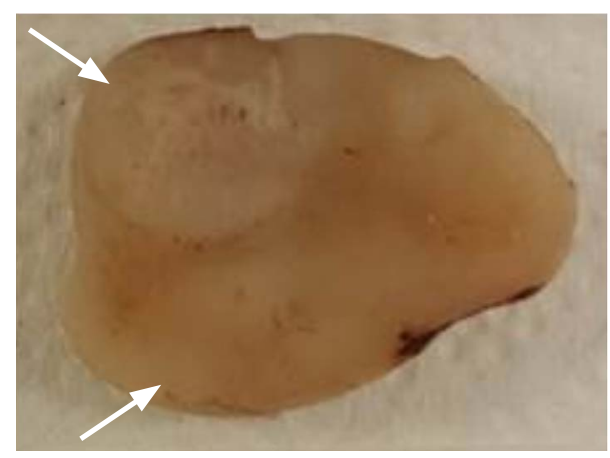

C

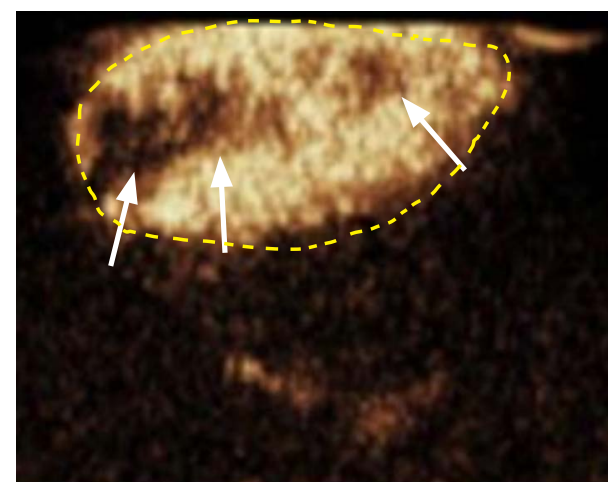

A

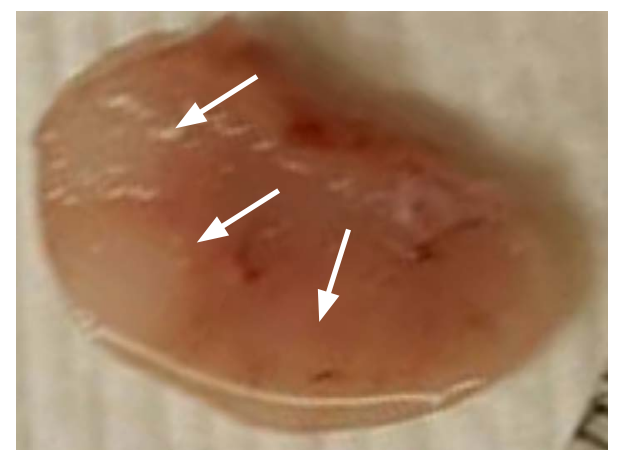

C

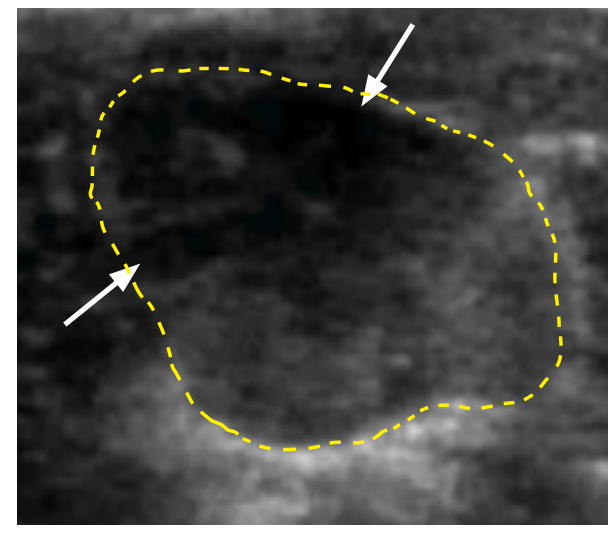

B

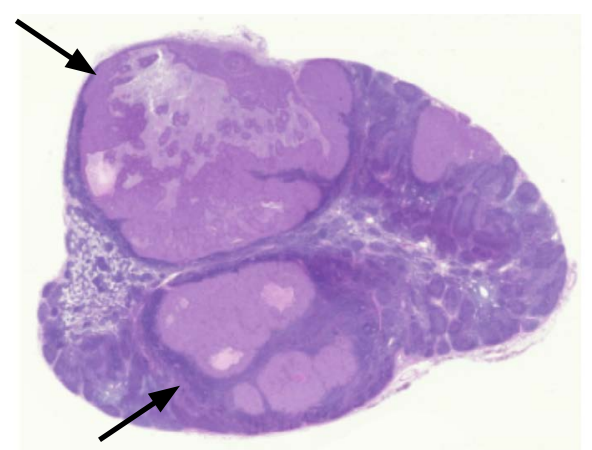

D

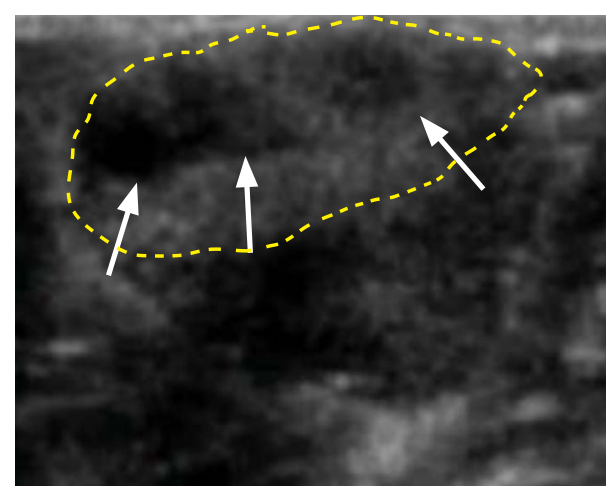

B

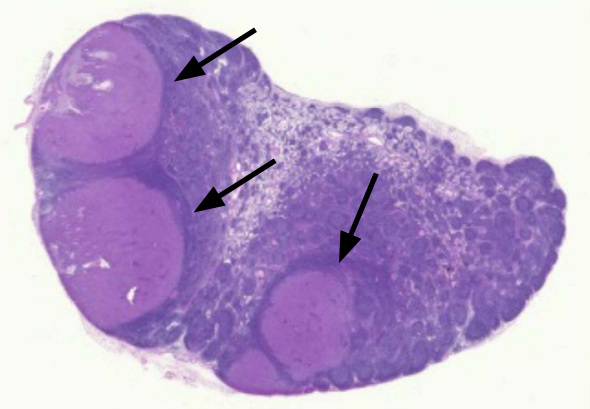

D
Fig. 3. Metastatic lymph node seen with lobulated perfusion defects on contrast-enhanced ultrasonography (CEUS) in group $A$.

$A, B$. CEUS (A) and gray-scale ultrasonography (B) show lobulated perfusion defects in a metastatic lymph node (arrows). C, D. A gross specimen (C) and a hematoxylin and eosinstained slide (D) show a lobulated metastatic tumor in the lymph node (arrows).
Fig. 4. Metastatic lymph node seen as multiple nodular perfusion defects on contrast-enhanced ultrasonography (CEUS) in group $B$.

A, B. CEUS (A) and gray-scale ultrasonography (B) show multiple nodular perfusion defects in a metastatic lymph node (arrows). C, D. A gross specimen (C) and a hematoxylin and eosin-stained slide (D) also show multiple tumor deposits in the lymph nodes (arrows). 


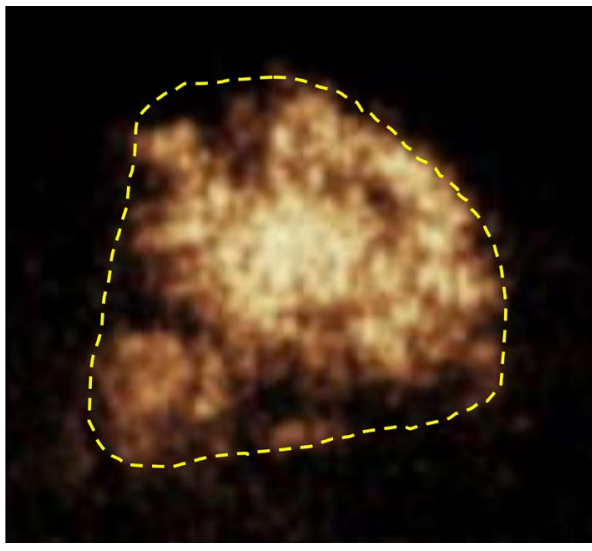

A

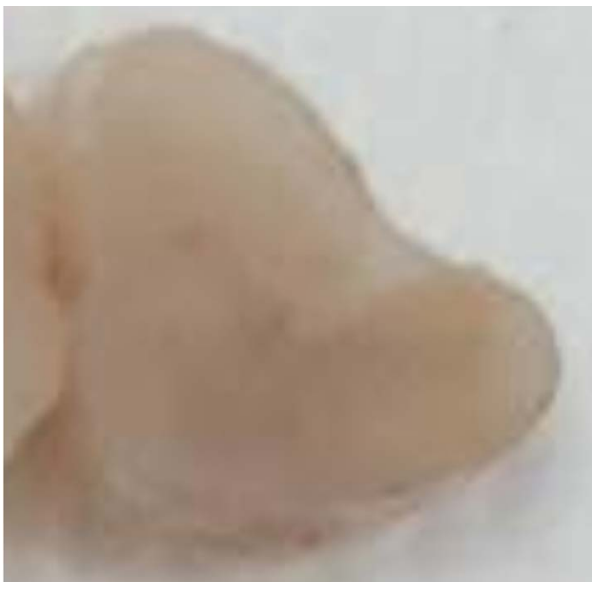

C

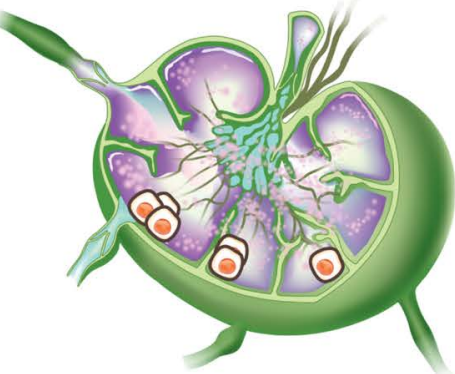

Initial stage

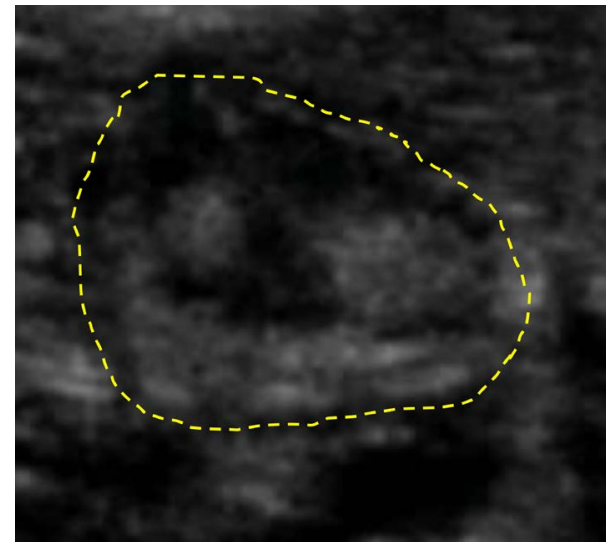

B

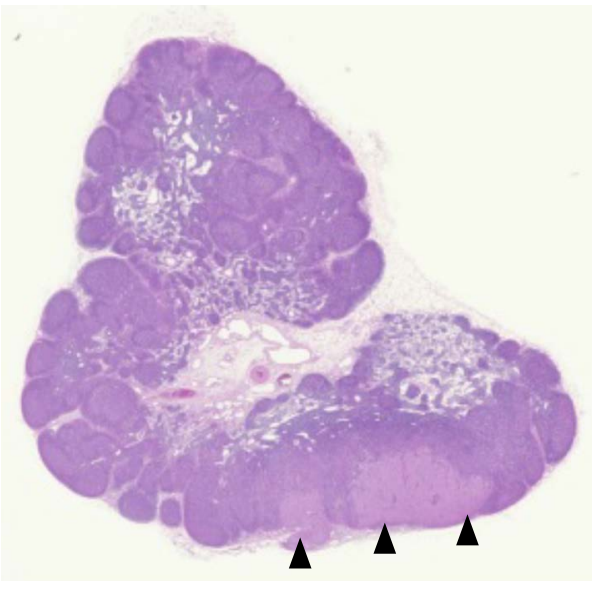

D

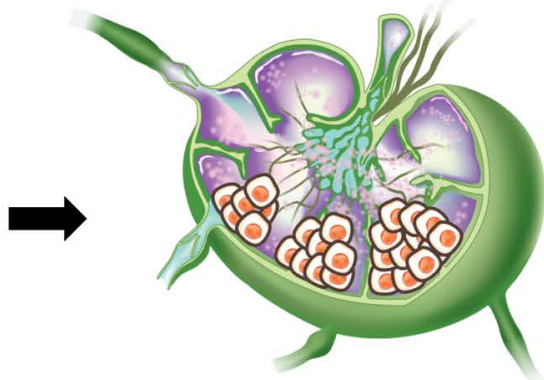

Growing stage
Fig. 5. Metastatic lymph node not seen on contrast-enhanced ultrasonography (CEUS) in group C.

$A, B$. CEUS (A) and gray-scale ultrasonography (B) show no definite perfusion defects. C, D. A gross specimen (C) and a hematoxylin and eosin-stained slide (D) show small areas of metastatic tumors (arrowheads) in the lymph node.

Fig. 6. Schematic drawings of the stages of lymph node metastasis. There are small perfusion defects in the peripheral portion of lymph node in the initial stage. Then, the metastatic areas grow into multiple nodular lesions. In the advanced stage, the multiple nodules are conglomerated and show lobulated contours.

round, filled defects revealing metastatic areas (Figs. 4, 6). Even though a perfusion defect was detected in all cases, 1 case (B-4 in Table 1) showed a discordant finding in comparison to the pathologic analysis. Group C was categorized as having a mean metastatic area of less than $1.0 \mathrm{~cm}^{2}$, and the mean percent area of metastasis was $9.1 \% \pm 6.4 \%$. The percent area of perfusion defects was $30.0 \% \pm 9.5 \%$. Only one case (C-1 in Table 1 ) showed a small perfusion defect on CEUS. Fig. 5 shows LNs with a minimal 
Table 1. Size of lymph nodes and tumors by group

\begin{tabular}{|c|c|c|c|c|c|c|c|}
\hline Group & Length $(\mathrm{cm})$ & Width (cm) & $\begin{array}{c}\text { Lymph node } \\
\text { dimensions }\left(\mathrm{cm}^{2}\right)\end{array}$ & $\begin{array}{c}\text { Tumor } \\
\text { dimensions }\left(\mathrm{cm}^{2}\right)\end{array}$ & $\begin{array}{l}\text { Area occupied by } \\
\text { the tumor }(\%)^{a)}\end{array}$ & $\begin{array}{c}\text { Perfusion defect } \\
(\%)^{\text {b) }}\end{array}$ & $\begin{array}{l}\text { CEUS/Pathology } \\
\text { concordance }^{c)}\end{array}$ \\
\hline$A-1$ & 1.90 & 1.38 & 1.90 & 0.87 & 45.60 & 58.58 & + \\
\hline$A-2$ & 2.20 & 1.02 & 2.11 & 1.39 & 66.00 & 83.99 & + \\
\hline$A-3$ & 1.85 & 1.70 & 3.02 & 0.74 & 24.40 & 69.33 & + \\
\hline A-4 & 2.00 & 1.60 & 2.62 & 0.70 & 26.80 & 79.63 & + \\
\hline Mean $\pm S D$ & $1.99 \pm 0.51$ & $1.42 \pm 0.32$ & $2.41 \pm 0.51$ & $0.92 \pm 0.32$ & $40.70 \pm 19.35$ & $74.48 \pm 9.82$ & \\
\hline$B-2$ & 1.40 & 0.75 & 1.18 & 0.43 & 36.60 & 47.15 & + \\
\hline B-3 & 1.50 & 1.14 & 1.74 & 0.32 & 18.60 & 43.14 & + \\
\hline B-4 & 1.13 & 1.00 & 1.87 & 0.06 & 2.90 & 39.89 & - \\
\hline Mean $\pm S D$ & $1.32 \pm 0.33$ & $0.92 \pm 0.17$ & $1.53 \pm 0.33$ & $0.29 \pm 0.17$ & $21.53 \pm 14.43$ & $42.34 \pm 9.69$ & \\
\hline$C-4$ & 1.16 & 0.90 & 0.69 & 0.01 & 13.00 & 38.98 & - \\
\hline Mean $\pm S D$ & $1.32 \pm 0.33$ & $0.92 \pm 0.17$ & $1.53 \pm 0.33$ & $0.29 \pm 0.17$ & $21.53 \pm 14.43$ & $30.02 \pm 9.54$ & \\
\hline P-value ${ }^{\text {d) }}$ & 0.019 & 0.073 & 0.007 & 0.010 & 0.049 & 0.007 & \\
\hline
\end{tabular}

SD, standard deviation.

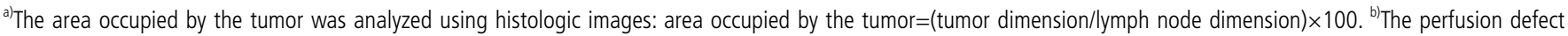
was calculated based on gray-scale and contrast-enhanced ultrasonography (CEUS) images, as follows: perfusion defect=[(area of gray image-area of CEUS image)/area of gray image $] \times 100$. 'Positive $(+)$ and negative $(-)$ signs represent identical or different diagnoses between the histologic and ultrasound analyses, respectively. ${ }^{d)} \mathrm{P}$-values were obtained using the Kruskal-Wallis test.

metastatic area. In all the groups, metastatic LNs with a perfusion defect area over $40 \%$ were positively matched with the pathologic results.

The dimensions of the metastatic LNs, the metastatic area of LNs, and the percent area of metastatic LNs among the three groups all showed statistically significant differences $(P=0.007, P=0.010$, and $\mathrm{P}=0.049$, respectively; Kruskal-Wallis test). The area of metastatic LNs was significantly different in all three groups ( $P=0.021$, MannWhitney $U$ test), between groups $A$ and $B(P=0.021$, Mann-Whitney $U$ test), and between groups $A$ and $C(P=0.020$, Mann-Whitney $U$ test). The percent area of metastatic LNs was significantly different between groups $A$ and $C(P=0.021$, Mann-Whitney $U$ test).

\section{Proposed Mechanism}

We visualized metastatic LNs on CEUS imaging and categorized them into three stages: the initial stage, the growth stage, and the advanced stage. Fig. 6 illustrates the gradual progression of LN metastasis that we propose. As illustrated by the LNs in group C, which had metastatic areas ranging from $9 \%$ to $20 \%$, in the initial stage in Fig. 6 , tiny and suspicious lesions are found in the peripheral portions of LNs. In group B, with a metastatic area ranging from $20 \%$ to $40 \%$, multiple nodular perfusion defects were noted, corresponding to the growth stage. In group $A$, with a metastatic area of over $40 \%$ (the most advanced cases in our study), multiple nodular lesions were conglomerated, corresponding to the advanced stage.

\section{Discussion}

SLNs, which are the first to receive lymphatic drainage from a cancer, are at the highest risk for regional metastasis $[3,9]$. The accurate detection and evaluation of SLNs are critical for diagnosing and staging cancer, estimating the prognosis, and deciding on a treatment plan [16-19].

Some methods exist for detecting SLNs intraoperatively. First, radio-colloid scintigraphy with intraoperative gamma probe counting or injection of blue dye is clinically useful in patients with melanoma and breast cancer; it is associated with a success rate of more than $95 \%$ and a failure rate of less than $3.4 \%$ in SLN identification [1926]. However, the conventional methods of SLN detection have some limitations. First, the limited spatial resolution of lymphoscintigraphy may pose difficulties in providing accurate anatomic information during a preoperative evaluation. Second, the intraoperative gammaprobe counting technique has inherent subjectivity in the detection 
method itself, as it requires a certain degree of operator experience with LN detection. Moreover, the high level of radioactivity at the primary injection site can hinder the detection of radioactive hot nodes by a gamma probe $[22-24,27-30]$.

In the last decade, researchers have focused on applying CEUS with MBs for this purpose [31-36]. Some reports have demonstrated consistent positive results regarding CEUS images of metastasis. Goldberg and colleagues described that uniformly increased echogenic SLNs did not contain metastases, whereas SLNs in areas that did not enhance had metastases [35]. Wang et al. [9] asserted that unenhanced areas of LNs may be related to abnormal lymphatic drainage, caused by factors such as tumor infiltration, proliferation, and chronic inflammation. Cox et al. [12] demonstrated the possibility of detecting SLNs in breast cancer patients using CEUS; only $2 \%$ of patients with an initial benign SLN core biopsy were found to have two or more LN metastases. Recognition of SLN metastases using conventional gray-scale US is difficult early in the process of diagnosis and treatment. Detection and recognition via CEUS may be helpful for deciding upon a therapeutic strategy, such as initiation of neoadjuvant chemotherapy [37]. However, those studies did not differentiate between partially-metastatic LNs and micro-metastatic LNs in CEUS findings.

There are two main limitations of our study. First, the number of animals was not enough to clearly detect and classify LNs by the combined technique of CEUS and MBs in the early stage and the growth stage with approximately $1-\mathrm{cm}$ LNs. To improve the sensitivity and specificity, we plan to conduct a further animal study. Second, because US examinations are intrinsically subjective, our results may reflect the radiologist's experience. A possible resolution may be for multiple radiologists to participate or to conduct a crossconfirmation using a secondary imaging modality or dye staining. However, SLN biopsy is the gold standard for patients with breast cancer and melanoma according to the National Comprehensive Cancer Network guideline.

In conclusion, we demonstrated that metastatic LNs could be successfully detected by CEUS imaging with a commercial contrast agent. Perfusion defects could be clearly detected in LNs with a metastatic area exceeding $40 \%$, and the CEUS images were compared with pathologic images to evaluate their correspondence. The high level of correspondence between the CEUS and pathologic images enabled not only the evaluation of metastatic LNs, but the determination of the stage of metastasis using CEUS imaging with a contrast agent. LN metastasis initially occurs as multiple peripheral lesions located in the peripheral area, then grows as multiple nodular filling defects, and finally becomes a conglomeration of filling defects on CEUS. Due to the wide accessibility of US imaging modality and real-time monitoring during surgery, we expect that
CEUS imaging will become a feasible alternative modality for evaluating LNs in the clinic.

ORCID: Shin-Woo Ha: https://orcid.org/0000-0001-5504-5255; Hyun-Je Lee: https:// orcid.org/0000-0001-7190-6043; Ae-Sin Cho: https://orcid.org/0000-0002-57630666; Sung II Hwang: https://orcid.org/0000-0001-7516-5369; Hak Jong Lee: https:// orcid.org/0000-0003-0858-7873

\section{Author Contributions}

Conceptualization: Lee HJ. Data acquisition: Cho AS, Lee HJ, Ha SW. Data analysis or interpretation: Ha SW, Hwang SI, Lee HJ. Drafting of the manuscript: Ha SW, Lee HJ. Critical revision of the manuscript Ha SW, Lee HJ. Approval of the final version of the manuscript: all authors.

\section{Conflict of Interest}

No potential conflict of interest relevant to this article was reported.

\section{Acknowledgments}

The authors thank Professor Jin-Haeng Chung of the Department of Pathology, Seoul National University Bundang Hospital for supervising the pathologic evaluation.

This research was supported by the Basic Science Research Program through the National Research Foundation of Korea (NRF) funded by the Ministry of Education (2015R1D1A1A09056744) and supported by a grant (D171751) from the Gyeonggi Technology Development Program funded by Gyeonggi Province.

\section{References}

1. Farnsworth RH, Achen MG, Stacker SA. The evolving role of lymphatics in cancer metastasis. Curr Opin Immunol 2018;53:6473.

2. Haffty BG, Yang Q, Reiss M, Kearney T, Higgins SA, Weidhaas J, et al. Locoregional relapse and distant metastasis in conservatively managed triple negative early-stage breast cancer. J Clin Oncol 2006:24:5652-5657.

3. Uren RF, Howman-Giles R, Chung D, Thompson JF. Imaging sentinel lymph nodes. Cancer J 2015;21:25-32.

4. Blomley MJ, Cooke JC, Unger EC, Monaghan MJ, Cosgrove DO. Microbubble contrast agents: a new era in ultrasound. BMJ 2001;322:1222-1225.

5. Goyal A. New technologies for sentinel lymph node detection. Breast Care (Basel) 2018;13:349-353.

6. Cui XW, Jenssen C, Saftoiu A, Ignee A, Dietrich CF. New ultrasound techniques for lymph node evaluation. World I Gastroenterol 2013;19:4850-4860.

7. Gramiak R, Shah PM. Echocardiography of the aortic root. Invest 
Radiol 1968;3:356-366.

8. Wang Y, Wang W, Li J, Tang J. Gray-scale contrast-enhanced ultrasonography of sentinel lymph nodes in a metastatic breast cancer model. Acad Radiol 2009;16:957-962.

9. Wang Y, Cheng Z, Li J, Tang J. Gray-scale contrast-enhanced ultrasonography in detecting sentinel lymph nodes: an animal study. Eur J Radiol 2010;74:e55-e59.

10. Qu E, Dai Z, Liang X, Qian Y, Wang $S$, Ke H, et al. Detection and pathologic evaluation of sentinel lymph nodes in the VX2 tumor model using a novel ultrasound/near-infrared dual-modality contrast agent. Ultrasound Med Biol 2015;41:1905-1912.

11. Nam K, Stanczak M, Forsberg F, Liu JB, Eisenbrey JR, Solomides $\mathrm{CC}$, et al. Sentinel lymph node characterization with a dualtargeted molecular ultrasound contrast agent. Mol Imaging Biol 2018;20:221-229.

12. Cox K, Weeks J, Mills P, Chalmers R, Devalia H, Fish D, et al. Contrast-enhanced ultrasound biopsy of sentinel lymph nodes in patients with breast cancer: implications for axillary metastases and conservation. Ann Surg Oncol 2016;23:58-64.

13. Kim KW, Lee JM, Kim JH, Klotz E, Kim HC, Han JK, et al. CT color mapping of the arterial enhancement fraction of VX2 carcinoma implanted in rabbit liver: comparison with perfusion CT. AJR Am J Roentgenol 2011;196:102-108.

14. Guo Y, Zhang Y, Jin N, Klein R, Nicolai J, Lewandowski RJ, et al. Electroporation-mediated transcatheter arterial chemoembolization in the rabbit VX2 liver tumor model. Invest Radiol 2012;47:116120.

15. Kim YI, Chung JW, Park JH, Han JK, Hong JW, Chung H. Intraarterial gene delivery in rabbit hepatic tumors: transfection with nonviral vector by using iodized oil emulsion. Radiology 2006;240:771-777.

16. Luke GP, Myers JN, Emelianov SY, Sokolov KV. Sentinel lymph node biopsy revisited: ultrasound-guided photoacoustic detection of micrometastases using molecularly targeted plasmonic nanosensors. Cancer Res 2014;74:5397-5408.

17. Gershenwald JE, Thompson W, Mansfield PF, Lee JE, Colome MI, Tseng $\mathrm{CH}$, et al. Multi-institutional melanoma lymphatic mapping experience: the prognostic value of sentinel lymph node status in 612 stage I or II melanoma patients. J Clin Oncol 1999;17:976983.

18. Stoeckli SJ, Steinert H, Pfaltz M, Schmid S. Sentinel lymph node evaluation in squamous cell carcinoma of the head and neck. Otolaryngol Head Neck Surg 2001;125:221-226.

19. Veronesi U, Paganelli G, Galimberti V, Viale G, Zurrida S, Bedoni $M$, et al. Sentinel-node biopsy to avoid axillary dissection in breast cancer with clinically negative lymph-nodes. Lancet 1997;349:1864-1867.

20. Lee YJ, Kim YH, Lee KH, Park JH, Lee HS, Jung SC, et al. Sentinel node mapping of VX2 carcinoma in rabbit thigh with CT lymphography using ethiodized oil. Korean J Radiol 2014;15:29-36.
21. Morton DL, Wen DR, Wong JH, Economou JS, Cagle LA, Storm FK, et al. Technical details of intraoperative lymphatic mapping for early stage melanoma. Arch Surg 1992;127:392-399.

22. Borgstein PJ, Pijpers R, Comans EF, van Diest PJ, Boom RP, Meijer S. Sentinel lymph node biopsy in breast cancer: guidelines and pitfalls of lymphoscintigraphy and gamma probe detection. J Am Coll Surg 1998; 186:275-283.

23. De Cicco C, Cremonesi M, Luini A, Bartolomei M, Grana C, Prisco $\mathrm{G}$, et al. Lymphoscintigraphy and radioguided biopsy of the sentinel axillary node in breast cancer. J Nucl Med 1998;39:2080-2084.

24. Linehan $D C$, Hill $A D$, Akhurst $T$, Yeung $H$, Yeh SD, Tran KN, et al. Intradermal radiocolloid and intraparenchymal blue dye injection optimize sentinel node identification in breast cancer patients. Ann Surg Oncol 1999;6:450-454.

25. Morton DL, Thompson JF, Cochran AJ, Mozzillo N, Elashoff R, Essner $\mathrm{R}$, et al. Sentinel-node biopsy or nodal observation in melanoma. N Engl J Med 2006;355:1307-1317.

26. Giuliano AE, Haigh PI, Brennan MB, Hansen NM, Kelley MC, Ye W, et al. Prospective observational study of sentinel lymphadenectomy without further axillary dissection in patients with sentinel nodenegative breast cancer. J Clin Oncol 2000;18:2553-2559.

27. Feldman SM, Krag DN, McNally RK, Moor BB, Weaver DL, Klein P. Limitation in gamma probe localization of the sentinel node in breast cancer patients with large excisional biopsy. J Am Coll Surg 1999; 188:248-254.

28. Dupont E, Cox C, Shivers S, Salud C, Nguyen K, Cantor A, et al. Learning curves and breast cancer lymphatic mapping: institutional volume index. J Surg Res 2001;97:92-96.

29. Mariani G, Moresco L, Viale G, Villa G, Bagnasco M, Canavese G, et al. Radioguided sentinel lymph node biopsy in breast cancer surgery. J Nucl Med 2001;42:1198-1215.

30. Tangoku A, Seike J, Nakano K, Nagao T, Honda J, Yoshida T, et al. Current status of sentinel lymph node navigation surgery in breast and gastrointestinal tract. J Med Invest 2007;54:1-18.

31. Wisner ER, Ferrara KW, Short RE, Ottoboni TB, Gabe JD, Patel D. Sentinel node detection using contrast-enhanced power Doppler ultrasound lymphography. Invest Radiol 2003;38:358-365.

32. Lurie DM, Seguin B, Schneider PD, Verstraete FJ, Wisner ER. Contrast-assisted ultrasound for sentinel lymph node detection in spontaneously arising canine head and neck tumors. Invest Radiol 2006;41:415-421.

33. Goldberg BB, Merton DA, Liu JB, Murphy G, Forsberg F. Contrastenhanced sonographic imaging of lymphatic channels and sentinel lymph nodes. J Ultrasound Med 2005;24:953-965.

34. Choi SH, Kono Y, Corbeil J, Lucidarme O, Mattrey RF. Model to quantify lymph node enhancement on indirect sonographic lymphography. AJR Am J Roentgenol 2004;183:513-517.

35. Goldberg BB, Merton DA, Liu JB, Thakur M, Murphy GF, Needleman $L$, et al. Sentinel lymph nodes in a swine model with melanoma: 
contrast-enhanced lymphatic US. Radiology 2004;230:727-734.

36. Omoto K, Hozumi Y, Omoto $Y$, Taniguchi N, Itoh K, Fujii Y, et al. Sentinel node detection in breast cancer using contrast-enhanced sonography with 25\% albumin: initial clinical experience. J Clin Ultrasound 2006;34:317-326.
37. Kilbride KE, Lee MC, Nees AV, Cimmino VM, Diehl KM, Sabel MS, et al. Axillary staging prior to neoadjuvant chemotherapy for breast cancer: predictors of recurrence. Ann Surg Oncol 2008;15:32523258. 